\title{
Erratum
}

\section{Phosphorylation of EBP50 negatively regulates $\beta$-PIX-dependent Rac1 activity in anoikis}

\author{
J-Y Chen ${ }^{1}$, Y-Y Lin ${ }^{1}$ and T-S Jou ${ }^{\star, 1,2,3}$ \\ ${ }^{1}$ Graduate Institute of Molecular Medicine, National Taiwan University, No.7, Chung-Shan S. Road, Taipei 100, Taiwan; ${ }^{2}$ Department of Internal Medicine, National \\ Taiwan, University Hospital and National Taiwan University College of Medicine, No.7, Chung-Shan S. Road, Taipei 100, Taiwan and ${ }^{3}$ Graduate Institute of Clinical \\ Medicine, National Taiwan University College of Medicine, No.7, Chung-Shan S. Road, Taipei 100, Taiwan \\ *Corresponding author: T-S Jou, Graduate Institute of Molecular Medicine, National Taiwan University, No. 7, Chung-Shan S. Road, Taipei 100, Taiwan. \\ Tel: + 8862 23123456, ext. 67625; Fax: + 8862 23709820; E-mail: jouts@ntu.edu.tw
}

Cell Death and Differentiation (2012) 19, 1092; doi:10.1038/cdd.2012.29

Correction to: Cell Death and Differentiation (2012) 19, 1027-1037; doi:10.1038/cdd.2012.4; published online 3 February 2012

Since the publication of this article, the authors have noticed that the address for affiliation 1 was incorrect.
This error has now been rectified. The correct address is shown above and the corrected article appears in this issue. The html and online pdf versions have also been rectified, and now with the correct affiliations.

We would like to apologize for any inconvenience this may have caused. 